\title{
Effect of levocarnitine administration in patients with chronic liver disease
}

\author{
KAZUKI OHASHI ${ }^{1}$, TORU ISHIKAWA ${ }^{2}$, ASAMI HOSHII ${ }^{3}$, TAMAKI HOKARI ${ }^{3}$, MITSUYUKI SUZUKI $^{4}$, \\ HIROHITO NOGUCHI $^{5}$, HIROSHI HIROSAWA ${ }^{6}$, FUJIKO KOYAMA ${ }^{5}$, MIKI KOBAYASHI ${ }^{5}$, \\ SHIORI HIROSAWA ${ }^{5}$, KAEDE SUGIYAMA ${ }^{7}$, YUTA MITOBE ${ }^{8}$ and TOSHIAKI YOSHIDA ${ }^{2}$ \\ ${ }^{1}$ Department of Nursing, Sapporo University of Health Sciences, Hokkaido, Sapporo 007-0894; \\ Departments of ${ }^{2}$ Gastroenterology and Hepatology, ${ }^{3}$ Medical Radiology, ${ }^{4}$ Pharmacology, ${ }^{5}$ Nursing, ${ }^{6}$ Clinical Engineering \\ and ${ }^{7}$ Nutrition, Saiseikai Niigata Hospital, Niigata 950-1104; ${ }^{8}$ Department of Nursing, \\ Teikyo University, Faculty of Medical Technology, Tokyo 173-0003, Japan
}

Received March 5, 2020; Accepted July 28, 2020

DOI: $10.3892 /$ etm.2020.9222

\begin{abstract}
L-carnitine administration was reported to improve sarcopenia in patients with cirrhosis. However, the amount of evidence from previous studies is not sufficient. The present study aimed to clarify the effect of levocarnitine (L-carnitine) administration on body composition in patients with chronic liver disease (CLD). In the present study, 85 patients with L-carnitine administration and 87 control patients were enrolled and divided them into two groups, the L-carnitine administration group ( $\mathrm{LAG}, \mathrm{n}=44$ ) and the without L-carnitine administration (controls, $n=44$ ) group, by using propensity score matching for age, sex, body mass index (BMI) and serum albumin. $\Delta$ skeletal muscle mass index (SMI)/year, $\Delta$ intramuscular adipose tissue content (IMAC)/year and $\Delta$ bone mineral density (BMD)/year were examined during L-carnitine administration. Each parameter was measured by computed tomography (CT) or dual-energy X-ray absorptiometry. The median age overall was 69 years (IQR, 64.0, 75.0); 36 were men and 52 were women. The median SMI was $37.4 \mathrm{~cm}^{2} / \mathrm{m}^{2}$ (IQR, 34.01, 44.34). The initial CT scans showed similar median values of SMI for the two groups [37.74 (34.17, 43.58) and 37.16 (33.83, 44.34), $\mathrm{P}=0.67]$. However, the median $\triangle \mathrm{SMI} /$ year for the LAG and controls
\end{abstract}

Correspondence to: Dr Toru Ishikawa, Department of Gastroenterology and Hepatology, Saiseikai Niigata Hospital, Teraji 280-7, Niigata 950-1104, Japan

E-mail: toruishi@ngt.saiseikai.or.jp

Abbreviations: CLD, chronic liver disease; DEXA, dual energy X-ray absorptiometry; FIB-4, fibrosis-4 index; HCC, hepatocellular carcinoma; IMAC, intramuscular adipose tissue content; L-carnitine, levocarnitine; LC, liver cirrhosis; LAG, L-carnitine administration group; LSBMD, lumbar spine bone mineral density; SMI, skeletal muscle mass index; WBBMD, whole body bone mineral density

Key words: sarcopenia, skeletal muscle, carnitine, bone mineral density, chronic liver disease were $0.95 \%(-3.07,6.10)$ and $-2.34 \%(-5.34,0.53)$, respectively $(\mathrm{P}=0.003)$. The median $\Delta$ whole body BMD/year for the LAG and controls were $-0.24 \%(-1.20,0.91)$ and $-1.04 \%(-2.16,0.47)$, respectively $(\mathrm{P}=0.038)$. The median $\triangle \mathrm{IMAC} /$ year and $\Delta$ lumbar spine BMD were not significantly different between the LAG and controls. L-carnitine administration may prevent the loss of skeletal muscle mass and BMD; therefore, it may be used as a new treatment option for osteoporosis and sarcopenia in patients with CLD.

\section{Introduction}

Sarcopenia is defined as a decrease in muscle strength and physical function and skeletal muscle mass depletion $(1,2)$. Primary sarcopenia is caused by aging; secondary sarcopenia is caused by malnutrition, sedentary behavior, and various clinical conditions, such as inflammatory disease, endocrine disease, and liver disease $(3,4)$. The prevalence of sarcopenia in patients with chronic liver disease (CLD) is ranged from 10 to $70 \%$ in Japan $(4,5)$. Recent studies have revealed that sarcopenia exacerbates survival, quality of life, and outcome after liver transplant in patients with liver cirrhosis (LC) (6-12). Since hepatocytes perform the function of glucose, lipid, and protein metabolism, liver dysfunction causes a glycogen storage dysfunction in the liver that facilitates the utilization of glycogen and branched amino acid from skeletal muscle, resulting in the progression of proteolysis $(13,14)$. Therefore, preventive treatment is needed to reduce the onset and progression of sarcopenia due to skeletal muscle depletion in patients with CLD.

Levocarnitine (L-carnitine) is an essential nutrient that plays a pivotal role in fatty acid metabolism (15). L-carnitine is involved in $\beta$-oxidation of fatty acids. It is a conditionally synthesized nutrient from amino acids methionine and lysine in the brain, liver, and kidney. Carnitine is obtained mainly from food; however, one-fourth of carnitine is synthesized in the kidney and liver $(14,16)$. In other words, L-carnitine deficiency occurs more frequently in patients with LC or CLD. Several reports about L-carnitine administration in patients with LC revealed that it improved muscle cramps, suppressed hepatic 
encephalopathy, and improved hyperammonemia $(17,18)$. Furthermore, L-carnitine administration may improve sarcopenia in patients with LC $(14,19)$. However, enough evidence has not been obtained in previous studies. Thus, this study aimed to clarify the effect of L-carnitine administration on body composition [skeletal muscle mass and bone mineral density (BMD)] in patients with CLD.

\section{Materials and methods}

Study design and patients. In this retrospective study, we reviewed 592 patients with CLD between 2015 and 2018 at Saiseikai Niigata Hospital. Of those patients, 85 were treated with L-carnitine (Otsuka Pharmaceutical, Tokyo, Japan) and underwent computed tomography (CT) twice during L-carnitine administration. The interval of CT scans was within 6-18 months. These 85 patients met the following inclusion criteria: i) Subsequent CT scans were conducted within 6-18 months that enabled evaluation of the change in skeletal muscle mass and clinical parameters and BMD by dual energy $\mathrm{X}$-ray; and ii) they continued L-carnitine administration during the observational period. Patients with hepatocellular carcinoma (HCC) were excluded (Fig. 1). In this study, the L-carnitine dose ranged from 1,500 to 3,000 $\mathrm{mg} /$ day, and the dose was selected by the attending physician. The reasons for L-carnitine administration were hepatic encephalopathy, muscle cramps, hyperammonemia, hypoalbuminemia, or combination of these conditions. We evaluated the effects of L-carnitine on body composition in patients with CLD. Moreover, 87 patients with CLD who did not receive L-carnitine and underwent paired CT scans to screen for HCC within 6-18 months were enrolled as controls. Cases (patients who received L-carnitine) and controls were matched for age, sex, body mass index (BMI), and serum albumin, using propensity score matching.

Evaluation for skeletal muscle mass and intramuscular adipose tissue content (IMAC). Skeletal muscle mass was evaluated using the skeletal muscle mass index (SMI) on CT scans. The SMI was calculated as follows: The sum of the cross-sectional area of skeletal muscles at the level of the third lumbar vertebra (L3) was measured by a radiological technologist using a region of interest (ROI) precisely traced with the use of commercially available image analysis software (volume analyzer SYNAPSE VINCENT, Fujifilm Medical Co., Ltd.), and this value was divided by height squared $\left(\mathrm{cm}^{2} / \mathrm{m}^{2}\right)$ (4). To evaluate the yearly change in SMI, $\Delta \mathrm{SMI} /$ year $(\%)$ was calculated as follows: $\Delta \mathrm{SMI} /$ year $(\%)=[(\mathrm{SMI}$ on the second CT-SMI on the initial CT)/SMI on the initial CT x100/interval between CT (day)/365] (19). CT was typically conducted in patients with CLD every 6-12 months according to the guidelines of the Japan Society of Hepatology (20).

Muscle quality was examined as IMAC at the L3 level. As previously described, IMAC was calculated by dividing the $\mathrm{CT}$ attenuation value of the multifidus muscles by that of the subcutaneous fat (21). To evaluate the yearly change in IMAC, $\triangle \mathrm{IMAC} /$ year was calculated as follows: $\triangle \mathrm{IMAC} /$ year $=[\mathrm{IMAC}$ on the second CT-IMAC on the initial $\mathrm{CT}] /[$ interval between CT (day)/365].
Evaluation of BMD. All patients underwent scanning of the total lumbar spine (L2-L4) BMD (LSBMD), and whole body BMD (WBBMD) by dual energy X-ray absorptiometry (DEXA) at each CT. To evaluate the yearly change in both BMD, $\triangle \mathrm{BMD} / \mathrm{year}(\%)$ was calculated as follows: $\triangle \mathrm{BMD} /$ year $(\%)=[(\mathrm{BMD}$ on the second DEXA-BMD on the initial DEXA)/BMD on the initial DEXA x100/interval between DEXA (day)/365].

Clinical and laboratory assessment. Patients underwent blood tests and CT on the same day. Clinical data were collected for the etiology of liver disease, BMI, and blood test results (white blood cells, platelet counts (Plt), serum albumin, aspartate aminotransferase (AST), alanine aminotransferase (ALT), cholinesterase, and hemoglobin A1c). The albumin-bilirubin (ALBI) score in each participant was calculated by the following formula as reported previously: ALBI score $=[\log 10$ total bilirubin $(\mu \mathrm{mol} / \mathrm{l}) \times 0.66]+$ [serum albumin $(\mathrm{g} / \mathrm{l}) \times-0.085$ ] while ALBI grade was classified into the following: ALBI score $\leq-2.60$, grade $1 ;-2.60<$ ALBI score $\leq-1.39$, grade 2 ; and ALBI score $>-1.39$, grade $3(22,23)$. The fibrosis-4 index (FIB-4) in each participant was calculated by the following formula as reported previously: FIB-4 = age (years) x AST (IU/l) $\div$ [platelets (109) x ALT (IU/l)] (24-26).

Statistical analysis. Continuous variables are presented as median and interquartile range (IQR) and analyzed using the Mann-Whitney U test. Categorical variables and nominal variables are presented as frequency (percentage) and analyzed using Fisher's exact test. We applied 1:1 propensity score matching to balance the assignment of patients with L-carnitine administration. The variables were age, sex, BMI, and serum albumin. Variables that affect sarcopenia were selected. Clinical features of CLD patients with sarcopenia were elderly, low BMI, and low albumin, and SMI depended on sex (27). Values of $\mathrm{P}<0.05$ were considered statistically significant. All statistical analyses were performed using EZR ver. 1.37 (Saitama Medical Center, Jichi Medical University, Saitama, Japan) (28).

\section{Results}

Patient characteristics and body composition at baseline and endpoint. We enrolled 85 patients with L-carnitine administration and 87 control patients in this study and divided these patients into two groups, namely, patients with L-carnitine administration (LAG, $n=44$ ) and patients without L-carnitine administration (controls, $\mathrm{n}=44$ ), by using propensity score matching for age, sex, BMI, and serum albumin. The overall characteristics (32 men and 56 women) are shown in Table I. The median age was 69 years (IQR, 64.0, 75.0). The median SMI was $37.4 \mathrm{~cm}^{2} / \mathrm{m}^{2}$ (IQR, 34.01,44.34). The etiology of CLD was hepatitis $B$ virus infection ( $n=10)$, hepatitis $C$ virus infection $(n=35)$, alcoholism $(n=8)$, nonalcoholic steatohepatitis and nonalcoholic fatty liver disease $(n=23)$, primary biliary cholangitis $(n=5)$, autoimmune hepatitis $(n=5)$, and others $(n=2)$. No significant difference in variables were found between the LAG and controls at baseline as well as at the endpoint (Table II). 


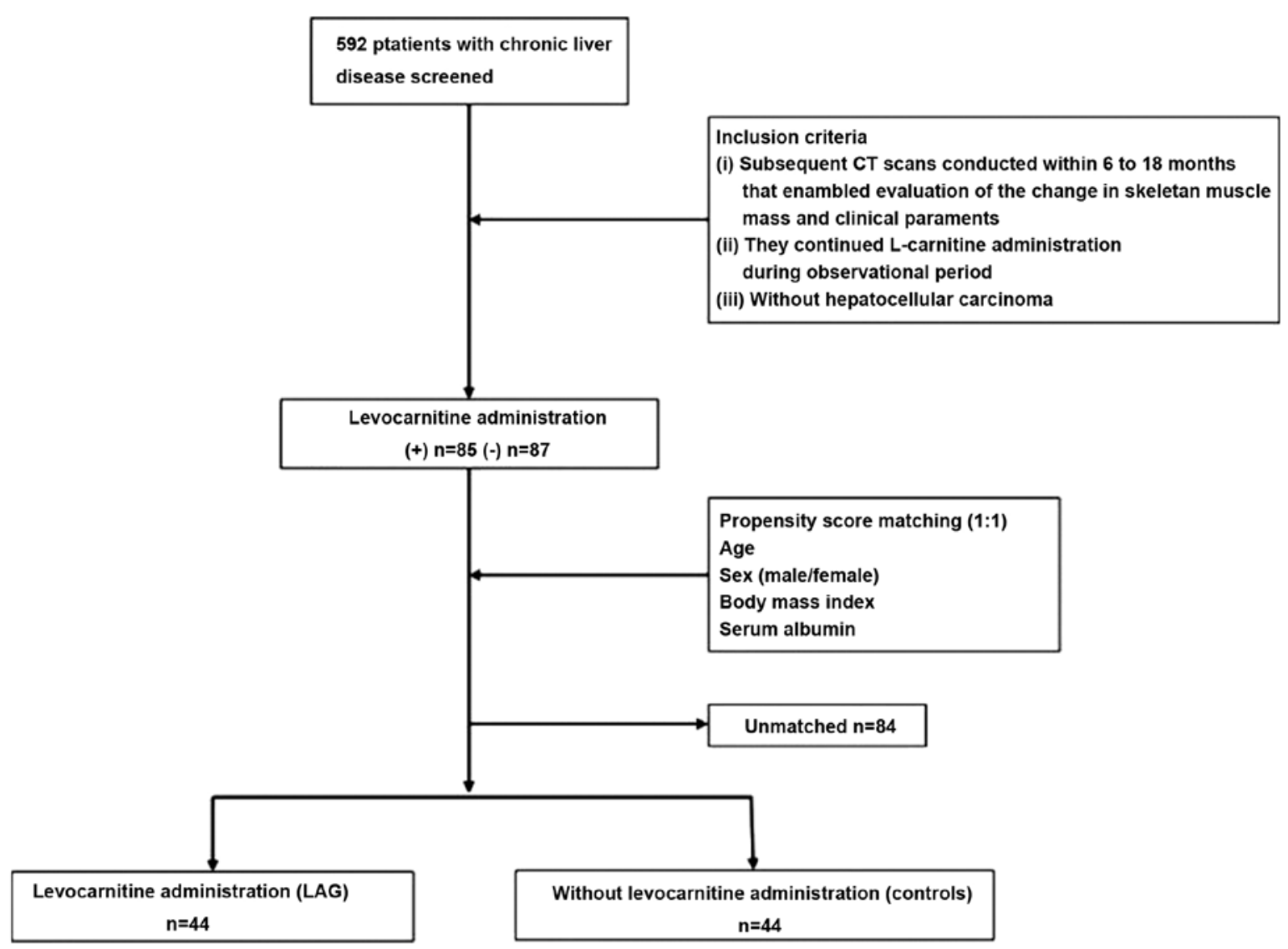

Figure 1. Patient flow chart. After screening 592 patients with chronic liver disease, 85 patients who were administered L-carnitine and 87 who were not administered L-carnitine were enrolled. After propensity score matching, 44 patients with L-carnitine administration were selected and 44 patients without L-carnitine administration were selected.

Comparison of change in SMI and body composition between the LAG and controls. The initial CT showed similar median values of SMI for the two groups $[37.74(34.17,43.58)$ and $37.16(33.83,44.34), \mathrm{P}=0.67]$. However, the median $\Delta \mathrm{SMI} /$ year for the LAG and controls were $0.95 \%(-3.07,6.10)$ and $-2.34 \%$ $(-5.34,0.53)$, respectively $(\mathrm{P}<0.01)$ (Fig. $2 \mathrm{~A})$. The median $\triangle \mathrm{IMAC} /$ year for the LAG and controls were $-0.00(-0.03,0.03)$ and $0.01(-0.02,0.04)$, respectively $(\mathrm{P}=0.46)$ (Fig. $2 \mathrm{~B})$. The median $\triangle \mathrm{WBBMD} / \mathrm{year}$ for the LAG and controls were $-0.24 \%(-1.20,0.91)$ and $-1.04 \%(-2.16,0.47)$, respectively $(\mathrm{P}=0.04)$ (Fig. 2C). The median $\triangle \mathrm{LSBMD} / \mathrm{year}$ for the $\mathrm{LAG}$ and controls were $-0.67 \%(-2.87,1.80)$ and $-0.18 \%(-1.99,2.99)$, respectively ( $\mathrm{P}=0.18)$ (Fig. 2D).

\section{Discussion}

Our results revealed that L-carnitine administration prevents skeletal muscle mass loss and osteoporosis. To our knowledge, this study is the first to report that L-carnitine may improve both sarcopenia and osteoporosis. Thus, the results of this study are of clinical significance for patients with CLD who have sarcopenia and osteoporosis.

The median $\Delta \mathrm{SMI} /$ year in our results was $0.69 \%$ for all patients, $0.95 \%$ for the LAG and $-2.34 \%$ for the controls. Especially, the median $\Delta \mathrm{SMI} / \mathrm{year}$ of the LAG was significantly better than that of the controls. According to previous studies, $\Delta \mathrm{SMI} /$ year or $\Delta$ skeletal muscle area/year of patients with LC ranged from -2.2 to $-0.22 \%(19,29)$. Our results show that skeletal muscle loss is suppressed compared with the results of these reports. In Japan, L-carnitine has been administered as a treatment for hepatic encephalopathy, hypoalbuminemia, and muscle cramps in patients with LC $(17,18)$. Our study includes patients with CLD who take L-carnitine for various purposes. However, the skeletal muscle mass of the LAG was significantly increased, suggesting that L-carnitine may prevent skeletal muscle mass loss.

There are multiple hypotheses that carnitine administration suppresses skeletal muscle loss in patients with LC. Carnitine plays a central role in transporting long-chain fatty acids from the cytosol to the mitochondrial matrix. Carnitine binds to the long-chain acyl coenzyme A and is converted to acylcarnitine. Acylcarnitine is transported to the mitochondria and degraded by $\beta$-oxidation $(14,30)$. Thus, carnitine administration improves energy metabolism disorders in the mitochondria in the liver (31). Improvement of these energy metabolism disorders is considered to suppress hyperammonemia in patients with LC (32). This is because the urea cycle, particularly localized in the liver, is a metabolic system that requires a lot of energy. Hyperammonemia activates myostatin and decreases muscle protein synthesis (33). Therefore, preventing hyperammonemia prevents skeletal muscle loss. Hiramatsu et al (19) reported that L-carnitine administration may suppress the progression of sarcopenia in conjunction with the improvement of hyperammonemia. The required dose of carnitine expected to inhibit 
Table I. Comparison of clinical and biochemical characteristics between the LAG and controls at baseline.

\begin{tabular}{|c|c|c|c|c|}
\hline Factor & All patients (IQR) & LAG (IQR) & Controls (IQR) & P-value \\
\hline Number of patients & 88 & 44 & 44 & \\
\hline Age (years) & $69.00(64.00,75.00)$ & $70.50(65.00,75.25)$ & $68.00(64.00,75.00)$ & 0.523 \\
\hline Sex & & & & 0.829 \\
\hline Male & 36 & 19 & 17 & \\
\hline Female & 52 & 25 & 27 & \\
\hline Body mass index $\left(\mathrm{kg} / \mathrm{m}^{2}\right)$ & $23.12(20.71,25.14)$ & $23.39(19.96,25.99)$ & $22.48(21.43,24.98)$ & 0.507 \\
\hline Skeletal muscle mass index $\left(\mathrm{cm}^{2} / \mathrm{m}^{2}\right)$ & $37.40(34.01,44.34)$ & $37.74(34.17,43.58)$ & $37.16(33.83,44.34)$ & 0.67 \\
\hline Intramuscular adipose tissue content & $-0.20(-0.30,-0.08)$ & $-0.22(-0.32,-0.10)$ & $-0.19(-0.27,-0.07)$ & 0.309 \\
\hline Visceral fat area $\left(\mathrm{cm}^{2}\right)$ & $99.00(61.79,137.82)$ & $90.47(53.98,125.77)$ & $109.06(77.08,137.82)$ & 0.313 \\
\hline Whole body bone mineral density $\left(\mathrm{g} / \mathrm{cm}^{2}\right)$ & $0.94(0.86,1.04)$ & $0.94(0.86,1.04)$ & $0.95(0.87,1.02)$ & 0.914 \\
\hline Lumber spine bone mineral density $\left(\mathrm{g} / \mathrm{cm}^{2}\right)$ & $0.87(0.75,1.07)$ & $0.87(0.74,1.05)$ & $0.87(0.78,1.09)$ & 0.599 \\
\hline Etiology & & & & 0.906 \\
\hline HBV & 10 & 4 & 6 & \\
\hline $\mathrm{HCV}$ & 35 & 17 & 18 & \\
\hline Alcohol & 8 & 4 & 4 & \\
\hline NASH and NAFLD & 23 & 12 & 11 & \\
\hline $\mathrm{PBC}$ & 5 & 4 & 1 & \\
\hline $\mathrm{AIH}$ & 5 & 2 & 3 & \\
\hline Other & 2 & 1 & 1 & \\
\hline ALBI score & $-2.89(-3.05,-2.70)$ & $-2.88(-3.05,-2.73)$ & $-2.90(-3.03,-2.68)$ & 0.877 \\
\hline ALBI grade $(1 / 2 / 3)$ & & & & 0.757 \\
\hline 1 & 76 & 37 & 39 & \\
\hline 2 & 12 & 7 & 5 & \\
\hline 3 & 0 & 0 & 0 & \\
\hline FIB-4 index & $2.30(1.66,3.14)$ & $2.62(1.82,3.51)$ & $2.23(1.58,2.74)$ & 0.123 \\
\hline White blood cells $\left(\times 10^{2} / \mu 1\right)$ & $55.00(46.00,62.25)$ & $55.50(49.75,63.50)$ & $53.50(44.75,61.00)$ & 0.251 \\
\hline Platelet counts $\left(\mathrm{x} 10^{4}\right)$ & $18.65(7.10,43.40)$ & $17.95(15.28,21.38)$ & $20.00(15.00,23.38)$ & 0.307 \\
\hline Albumin $(\mathrm{g} / \mathrm{dl})$ & $4.10(3.98,4.32)$ & $4.15(4.00,4.30)$ & $4.10(3.90,4.40)$ & 0.943 \\
\hline Aspartate aminotransferase (U/l) & $25.00(21.00,30.00)$ & $25.00(21.00,31.50)$ & $24.50(19.00,30.00)$ & 0.435 \\
\hline Alanine aminotransferase (U/l) & $17.00(12.00,25.00)$ & $17.00(12.00,23.50)$ & $17.00(11.00,25.00)$ & 0.947 \\
\hline Cholinesterase (U/l) & $297.00(247.75,340.00)$ & $282.00(237.75,332.25)$ & $297.50(266.50,353.25)$ & 0.346 \\
\hline Triglyceride (mg/dl) & $101.00(73.00,124.75)$ & $107.50(71.75,143.75)$ & $93.00(73.25,113.00)$ & 0.335 \\
\hline$\gamma-\mathrm{GTP}(\mathrm{IU} / 1)$ & $17.50(13.00,32.75)$ & $18.00(13.00,35.50)$ & $17.00(1300,30.50)$ & 0.904 \\
\hline Total cholesterol (mg/dl) & $186.00(161.25,212.00)$ & $184.00(161.75,212.25)$ & $186.50(161.00,210.00)$ & 0.893 \\
\hline Hemoglobin A1c (\%) & $5.80(5.50,6.20)$ & $5.80(5.45,6.20)$ & $5.80(5.53,6.10)$ & 0.711 \\
\hline Observational period (year) & $0.98(0.85,1.10)$ & $0.92(0.74,1.02)$ & $0.99(0.90,1.23)$ & 0.007 \\
\hline
\end{tabular}

Data were presented as number of patients or median (interquartile range). LAG, Levocarnitine administration group; HBV, hepatitis B virus; $\mathrm{HCV}$, hepatitis $\mathrm{C}$ virus; NASH, nonalcoholic steato-hepatitis; NAFLD, nonalcoholic fatty liver disease; PBC, primary biliary cholangitis; AIH, autoimmune hepatitis; FIB-4, fibrosis-4; IQR, interquartile range; $\gamma$-GTP, glutamyl transpeptidase; ALBI, ALBI, albumin-bilirubin.

the progression of sarcopenia is $\geq 1,274 \mathrm{mg} /$ day (19), and the carnitine dose administered in this study (1,500-3,000 mg/day) exceeded that. However, since this is a retrospective study, serum ammonia was not measured in many cases. Prospective studies are needed to clarify the relationship between formed ammonia levels and skeletal muscle mass.

To date, carnitine administration has been suggested to increase lipid utilization in the skeletal muscle during low exercise and improve exercise performance (34). We considered that carnitine not only increased skeletal muscle mass but also improved the quality and examined changes in IMAC, the quality indicator of skeletal muscle (21), but did not yield significant results in our study. Studies reported that fatty infiltration of the muscle (myosteatosis) exacerbates hepatic encephalopathy in LC (35) and is a prognostic factor for liver transplant patients $(21,36)$. Therefore, there is a need for treatments that improve not only skeletal muscle mass but also muscle quality. However, since the exercise and activity levels are not managed in this study, the effect of carnitine on IMAC may be unclear. In the future, a combination of carnitine and exercise may improve skeletal muscle quality. 
Table II. Comparison of clinical and biochemical characteristics between the LAG and controls at endpoint.

\begin{tabular}{|c|c|c|c|c|}
\hline Factor & All patients (IQR) & LAG (IQR) & Controls (IQR) & P-value \\
\hline Number of patients & 88 & 44 & 44 & \\
\hline Skeletal muscle mass index $\left(\mathrm{cm}^{2} / \mathrm{m}^{2}\right)$ & $37.99(32.98,44.08)$ & $39.58(34.63,44.46)$ & $36.15(32.43,43.49)$ & 0.166 \\
\hline Intramuscular adipose tissue content & $-0.20(-0.30,-0.07)$ & $-0.22(-0.33,-0.08)$ & $-0.19(-0.26,-0.08)$ & 0.367 \\
\hline Visceral fat area $\left(\mathrm{cm}^{2}\right)$ & $102.48(62.22,137.26)$ & $92.38(58.48,147.07)$ & $114.33(66.44,135.65)$ & 0.404 \\
\hline All bone mineral density $\left(\mathrm{g} / \mathrm{cm}^{2}\right)$ & $0.93(0.85,1.03)$ & $0.93(0.86,1.04)$ & $0.93(0.85,1.02)$ & 0.764 \\
\hline LS bone mineral density $\left(\mathrm{g} / \mathrm{cm}^{2}\right)$ & $0.87(0.76,1.08)$ & $0.86(0.74,1.05)$ & $0.87(0.78,1.09)$ & 0.384 \\
\hline ALBI score & $-2.89(-3.07,-2.73)$ & $-2.83(-3.05,-2.69)$ & $-2.94(-3.09,-2.77)$ & 0.206 \\
\hline ALBI grade & & & & 0.352 \\
\hline 1 & 76 & 36 & 40 & \\
\hline 2 & 12 & 8 & 4 & \\
\hline 3 & 0 & 0 & 0 & \\
\hline FIB-4 index & $2.20(1.66,2.88)$ & $2.50(1.88,2.95)$ & $2.12(1.45,2.73)$ & 0.115 \\
\hline White blood cells $\left(\mathrm{x} 10^{2} / \mu \mathrm{l}\right)$ & $58.00(44.00,70.25)$ & $57.00(42.75,68.00)$ & $59.00(44.75,70.50)$ & 0.907 \\
\hline Platelet counts $\left(\mathrm{x} 10^{4}\right)$ & $19.65(16.17,23.30)$ & $19.10(15.35,21.77)$ & $20.20(17.38,25.72)$ & 0.139 \\
\hline Albumin $(\mathrm{g} / \mathrm{dl})$ & $4.20(4.00,4.40)$ & $4.10(3.90,4.32)$ & $4.25(4.00,4.40)$ & 0.242 \\
\hline Aspartate aminotransferase (U/l) & $24.00(20.75,29.25)$ & $24.50(21.00,29.25)$ & $23.50(19.75,28.00)$ & 0.256 \\
\hline Alanine aminotransferase (U/1) & $16.00(12.00,22.25)$ & $17.00(13.00,23.25)$ & $15.00(10.75,21.25)$ & 0.171 \\
\hline Cholinesterase (U/l) & $288.50(250.50,350.25)$ & $282.00(221.75,332.50)$ & $293.00(254.75,351.25)$ & 0.138 \\
\hline Triglyceride (mg/dl) & $94.00(72.00,123.75)$ & $106.00(72.00,126.00)$ & $92.00(71.75,121.75)$ & 0.349 \\
\hline$\gamma-\mathrm{GTP}(\mathrm{IU} / \mathrm{l})$ & $16.00(12.00,32.00)$ & $16.50(12.75,29.00)$ & $16.00(12.00,32.25)$ & 0.691 \\
\hline Total cholesterol (mg/dl) & $180.00(161.25,206.75)$ & $179.00(162.75,203.25)$ & $181.00(160.25,212.00)$ & 0.99 \\
\hline Hemoglobin A1c (\%) & $5.80(5.50,6.25)$ & $5.80(5.50,6.30)$ & $5.80(5.70,6.10)$ & 0.971 \\
\hline
\end{tabular}

Data were presented as number of patients or median (interquartile range). LAG, Levocarnitine administration group; FIB-4, fibrosis-4; IQR, interquartile range; $\gamma$-GTP, glutamyl transpeptidase; ALBI, albumin-bilirubin; LS, lumbar spine.

A

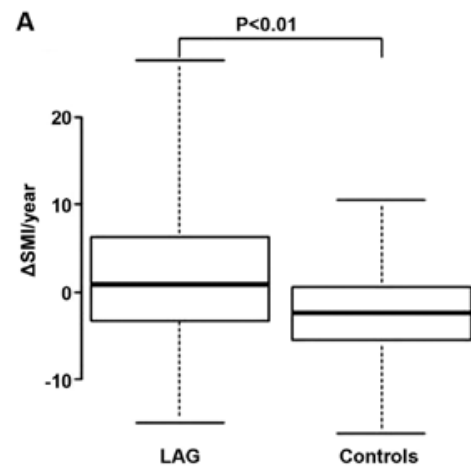

C

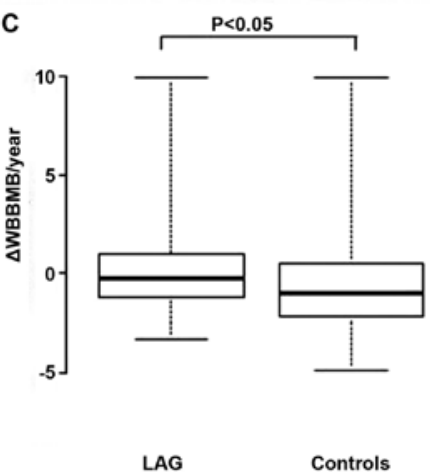

B

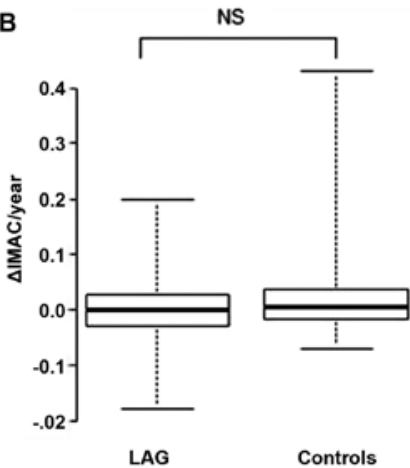

D

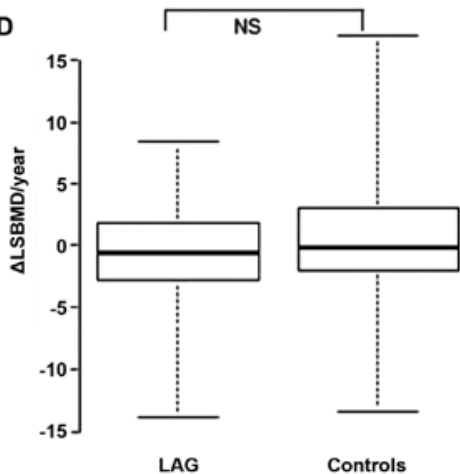

Figure 2. Comparison of $\triangle \mathrm{SMI} /$ year, $\triangle \mathrm{IMAC} / \mathrm{year}, \triangle \mathrm{WBBMD} / \mathrm{year}$, and $\triangle \mathrm{LSBMD}$ in the LAG and controls. (A) Comparison of $\triangle \mathrm{SMI} / \mathrm{year}$ in the LAG and controls. (B) Comparison of $\triangle \mathrm{IMAC} /$ year in the LAG and controls. (C) Comparison of $\triangle \mathrm{WBBMD} /$ year in the LAG and controls. (D) Comparison of $\triangle \mathrm{LSBMD}$ in the LAG and controls. Data were analyzed with the Mann-Whitney U test. Values of P<0.05 were considered statistically significant. LAG, levocarnitine administration group; IMAC, intramuscular adipose tissue content; NS, not significant; SMI, skeletal muscle mass index; IMAC, intramuscular adipose tissue content; WBBMD, whole body bone mineral density; LSBMD, lumbar spine bone mineral density. 
Furthermore, our study suggested that carnitine administration may prevent BMD loss. The prevalence of osteoporosis in patients with LC cirrhosis is $\sim 12-55 \%$, which is higher than in healthy individuals (37). Along with sarcopenia, it is one of the issues that have a major influence on the health of patients with CLD. One of the mechanisms of osteoporosis in patients with CLD has been shown to activate osteoclasts by inflammatory cytokines (38). Because carnitine suppresses the production of inflammatory cytokines (39), it may have prevented the decrease in $\triangle \mathrm{WBBMD}$ in our study. However, $\triangle$ LSBMD showed no significant effect. Further research is needed to treat osteoporosis in patients with CLD.

This study had several limitations. First, this was a retrospective single-center study with a small sample size. Moreover, the observation period was different for each patient. Second, the purpose and dose of L-carnitine administration were decided by the attending physician. Thus, prospective studies are warranted to clarify the effects of L-carnitine on preventing skeletal muscle loss in patients with liver disease.

In conclusion, we showed that L-carnitine administration prevented the loss of skeletal muscle mass and BMD in patients with CLD. L-carnitine administration can be a new option for treating osteoporosis and sarcopenia. Further detailed studies are needed to confirm this possibility.

\section{Acknowledgements}

Not applicable.

\section{Funding}

No funding was received.

\section{Availability of data and materials}

All data generated or analyzed in this study are included in this published article.

\section{Authors' contributions}

$\mathrm{KO}$ and TI designed the research; TI and TY conducted the research; AH, TH evaluated SMI, IMAC and BMD. MS collected the medication data. HN, HH, FK, MK, SH, and KS collected the clinical and laboratory assessment data; KO, TI, and YM analyzed the data; KO summarized the data; $\mathrm{KO}$ and TI wrote the manuscript. All authors have read, checked, and approved the final manuscript.

\section{Ethics approval and consent to participate}

The present study was conducted in accordance with the Declaration of Helsinki. Ethical approval was obtained from the ethics committees of Saiseikai Niigata Hospital (approval no. E17-27). Informed consent was obtained by the opt-out method on the website.

\section{Patient consent for publication}

Not applicable.

\section{Competing interests}

The authors declare that they have no competing interests.

\section{References}

1. Cruz-Jentoft AJ, Landi F, Schneider SM, Zúñiga C, Arai H, Boirie Y, Chen LK, Fielding RA, Martin FC, Michel JP, et al: Prevalence of and interventions for sarcopenia in ageing adults: A systematic review. Report of the International Sarcopenia Initiative (EWGSOP and IWGS). Age Ageing 43: 748-759, 2014.

2. Chen LK, Liu LK, Woo J, Assantachai P, Auyeung TW, Bahyah KS, Chou MY, Chou LY, Hsu PS, Krairit O, et al: Sarcopenia in Asia: Consensus report of the Asian working group for sarcopenia. J Am Med Dir Assoc 15: 95-101, 2014.

3. Cruz-Jentoft AJ, Baeyens JP, Bauer JM, Boirie Y, Cederholm T, Landi F, Martin FC, Michel JP, Rolland Y, Schneider SM, et al: Sarcopenia: European consensus on definition and diagnosis: Report of the European working group on Sarcopenia in older people. Age Ageing 39: 412-423, 2010.

4. Nishikawa H, Shiraki M, Hiramatsu A, Moriya K, Hino K and Nishiguchi S: Japan Society of Hepatology guidelines for sarcopenia in liver disease (1st edition): Recommendation from the working group for creation of sarcopenia assessment criteria. Hepatol Res 46: 951-963, 2016.

5. Ohashi K, Ishikawa T, Hoshi A, Suzuki M, Mitobe Y, Yamada E, Abeywickrama HM, Seki N, Koyama C, Aoki H and Koyama Y: Relationship between sarcopenia and both physical activity and lifestyle in patients with chronic liver disease. J Clin Med Res 10: 920-927, 2018.

6. Hanai T, Shiraki M, Nishimura K, Ohnishi S, Imai K, Suetsugu A, Takai K, Shimizu M and Moriwaki H: Sarcopenia impairs prognosis of patients with liver cirrhosis. Nutrition 31: 193-199, 2015.

7. Iritani S, Imai K, Takai K, Hanai T, Ideta T, Miyazaki T, Suetsugu A, Shiraki M and Moriwaki H: Skeletal muscle depletion is an independent prognostic factor for hepatocellular carcinoma. J Gastroenterol 50: 323-332, 2015.

8. Kim G, Kang SH, Kim MY and Baik SK: Prognostic value of sarcopenia in patients with liver cirrhosis: A systematic review and meta-analysis. PLoS One 12: e0186990, 2017.

9. Hamaguchi Y, Kaido T, Okumura S, Kobayashi A, Hammad A, Tamai Y, Inagaki N and Uemoto S: Proposal for new diagnostic criteria for low skeletal muscle mass based on computed tomography imaging in Asian adults. Nutrition 32: 1200-1205, 2016.

10. Kaido T, Ogawa K, Fujimoto Y, Ogura Y, Hata K, Ito T, Tomiyama K, Yagi S, Mori A and Uemoto S: Impact of sarcopenia on survival in patients undergoing living donor liver transplantation. Am J Transplant 13: 1549-1556, 2013.

11. Fujiwara N, Nakagawa H, Kudo Y, Tateishi R, Taguri M, Watadani T, Nakagomi R, Kondo M, Nakatsuka T, Minami T, et al: Sarcopenia, intramuscular fat deposition, and visceral adiposity independently predict the outcomes of hepatocellular carcinoma. J Hepatol 63: 131-140, 2015.

12. Ohashi K, Ishikawa T, Imai M, Suzuki M, Hoshii A, Abe H, Koyama F, Nakano T, Ueki A, Noguchi H, et al: Relationship between pre-sarcopenia and quality of life in patients with chronic liver disease: A cross-sectional study. Eur J Gastroenterol Hepatol 31: 1408-1413, 2019.

13. Moriwaki H, Miwa Y, Tajika M, Kato M, Fukushima $\mathrm{H}$ and Shiraki M: Branched-chain amino acids as a protein- and energy-source in liver cirrhosis. Biochem Biophys Res Commun 313: 405-409, 2004.

14. Ohara M, Ogawa K, Suda G, Kimura M, Maehara O, Shimazaki T, Suzuki K, Nakamura A, Umemura M, Izumi T, et al: L-carnitine suppresses loss of skeletal muscle mass in patients with liver cirrhosis. Hepatol Commun 2: 906-918, 2018.

15. Pekala J, Patkowska-Sokoła B, Bodkowski R, Jamroz D, Nowakowski P, Lochyński S and Librowski T: L-carnitinemetabolic functions and meaning in humans life. Curr Drug Metab 12: 667-678, 2011.

16. Kendler BS: Carnitine: An overview of its role in preventive medicine. Prev Med (Baltim) 15: 373-390, 1986.

17. Nakanishi H, Kurosaki M, Tsuchiya K, Nakakuki N, Takada H, Matsuda S, Gondo K, Asano Y, Hattori N, Tamaki N, et al: L-carnitine reduces muscle cramps in patients with cirrhosis. Clin Gastroenterol Hepatol 13: 1540-1543, 2015. 
18. Shiraki M, Shimizu M, Moriwaki H, Okita K and Koike K: Carnitine dynamics and their effects on hyperammonemia in cirrhotic Japanese patients. Hepatol Res 47: 321-327, 2017.

19. Hiramatsu A, Aikata H, Uchikawa S, Ohya K, Kodama K, Nishida Y, Daijo K, Osawa M, Teraoka Y, Honda F, et al: Levocarnitine use is associated with improvement in sarcopenia in patients with liver cirrhosis. Hepatol Commun 3: 348-355, 2019.

20. Clinical practice guidelines for hepatocellular carcinoma differ between Japan, United States, and Europe. Liver Cancer 4: 85-95, 2015.

21. Hamaguchi Y, Kaido T, Okumura S, Fujimoto Y, Ogawa K, Mori A, Hammad A, Tamai Y, Inagaki N and Uemoto S: Impact of quality as well as quantity of skeletal muscle on outcomes after liver transplantation. Liver Transpl 20: 1413-1419, 2014.

22. Johnson PJ, Berhane S, Kagebayashi C, Satomura S, Teng M, Reeves HL, O'Beirne J, Fox R, Skowronska A, Palmer D, et al: Assessment of liver function in patients with hepatocellular carcinoma: A new evidence-based approach-the ALBI grade. J Clin Oncol 33: 550-558, 2015.

23. Hiraoka A, Kumada T, Kudo M, Hirooka M, Tsuji K, Itobayashi E, Kariyama K, Ishikawa T, Tajiri K, Ochi $\mathrm{H}$, et al: Albumin-Bilirubin (ALBI) grade as part of the evidence-based clinical practice guideline for HCC of the Japan Society of Hepatology: A comparison with the liver damage and child-pugh classifications. Liver Cancer 6: 204-215, 2017.

24. Vallet-Pichard A, Mallet V, Nalpas B, Verkarre V, Nalpas A, Dhalluin-Venier V, Fontaine H and Pol S: FIB-4: An inexpensive and accurate marker of fibrosis in HCV infection. Comparison with liver biopsy and fibrotest. Hepatology 46 : 32-36, 2007.

25. Sumida Y, Yoneda M, Hyogo H, Itoh Y, Ono M, Fujii H, Eguchi Y, Suzuki Y, Aoki N, Kaneyama K, et al: Validation of the FIB4 index in a Japanese nonalcoholic fatty liver disease population. BMC Gastroenterol 12: 2, 2012.

26. Sterling RK, Lissen E, Clumeck N, Sola R, Correa MC, Montaner J, Sulkowski MS, Torriani FJ, Dieterich DT, Thomas DL, et al: Development of a simple noninvasive index to predict significant fibrosis in patients with $\mathrm{HIV} / \mathrm{HCV}$ coinfection. Hepatology 43: 1317-1325, 2006.

27. Nishikawa H, Enomoto H, Yoh K, Iwata Y, Sakai Y, Kishino K, Ikeda N, Takashima T, Aizawa N, Takata R, et al: Association between sarcopenia and depression in patients with chronic liver diseases. J Clin Med 8: 634, 2019.

28. Kanda Y: Investigation of the freely available easy-to-use software 'EZR' for medical statistics. Bone Marrow Transplant 48: 452-458, 2013.
29. Hanai T, Shiraki M, Ohnishi S, Miyazaki T, Ideta T, Kochi T, Imai K, Suetsugu A, Takai K, Moriwaki H and Shimizu M: Rapid skeletal muscle wasting predicts worse survival in patients with liver cirrhosis. Hepatol Res 46: 743-751, 2016.

30. Flanagan JL, Simmons PA, Vehige J, Willcox MD and Garrett Q: Role of carnitine in disease. Nutr Metab (Lond) 7: 30, 2010.

31. Sakai Y, Nishikawa H, Enomoto H, Yoh K, Iwata Y, Hasegawa K, Nakano C, Kishino K, Shimono Y, Takata R, et al: Effect of L-carnitine in patients with liver cirrhosis on energy metabolism using indirect calorimetry: A pilot study. J Clin Med Res 8: 863-869, 2016

32. Malaguarnera M, Pistone G, Elvira R, Leotta C, Scarpello L and Liborio R: Effects of L-carnitine in patients with hepatic encephalopathy. World J Gastroenterol 11: 7197-7202, 2005

33. Qiu J, Thapaliya S, Runkana A, Yang Y, Tsien C, Mohan ML, Narayanan A, Eghtesad B, Mozdzak PE, McDobald C, et al: Hyperammonemia in cirrhosis induces transcriptional regulation of myostatin by an NF- $\kappa \mathrm{B}-$ mediated mechanism. Proc Natl Acad Sci USA 110: 18162-18167, 2013.

34. Sawicka AK, Hartmane D, Lipinska P, Wojtowicz E, Lysiak-Szydlowska W and Olek RA: L-carnitine supplementation in older women. A pilot study on aging skeletal muscle mass and function. Nutrients 10: 255, 2018

35. Bhanji RA, Moctezuma-Velazquez C, Duarte-Rojo A, Ebadi M, Ghosh S, Rose C and Montano-Loza AJ: Myosteatosis and sarcopenia are associated with hepatic encephalopathy in patients with cirrhosis. Hepatol Int 12: 377-386. 2018.

36. Hamaguchi Y, Kaido T, Okumura S, Kobayashi A, Shirai H, Yao S, Yagi S, Kamo N, Okajima H and Uemoto S: Proposal for new selection criteria considering pre-transplant muscularity and visceral adiposity in living donor liver transplantation. J Cachexia Sarcopenia Muscle 9: 246-254, 2018.

37. Patel $\mathrm{N}$ and Muñoz SJ: Bone disease in cirrhosis. Clin Liver Dis (Hoboken) 6: 96-99, 2015.

38. Tilg H, Moschen AR, Kaser A, Pines A and Dotan I: Gut, inflammation and osteoporosis: Basic and clinical concepts. Gut 57: 684-694, 2008.

39. Shakeri A, Tabibi $\mathrm{H}$ and Hedayati M: Effects of L-carnitine supplement on serum inflammatory cytokines, C-reactive protein, lipoprotein (a), and oxidative stress in hemodialysis patients with Lp (a) hyperlipoproteinemia. Hemodial Int 14: 498-504, 2010.

This work is licensed under a Creative Commons Attribution-NonCommercial-NoDerivatives 4.0 International (CC BY-NC-ND 4.0) License. 